\title{
The Zero Active Mass Condition in Friedmann-Robertson-Walker Cosmologies
}

\author{
F. Melia* \\ Department of Physics, The Applied Math Program, \\ and Department of Astronomy, The University of Arizona, AZ 85721, USA
}

\begin{abstract}
Many cosmological measurements today suggest that the Universe is expanding at a constant rate. This is inferred from the observed age versus redshift relationship and various distance indicators, all of which point to a cosmic equation of state (EoS) $p=-\rho / 3$, where $\rho$ and $p$ are, respectively, the total energy density and pressure of the cosmic fluid. It has recently been shown that this result is not a coincidence and simply confirms the fact that the symmetries in the FriedmannRobertson-Walker (FRW) metric appear to be viable only for a medium with zero active mass, i.e., $\rho+3 p=0$. In their latest paper, however, Kim, Lasenby and Hobson have provided what they believe to be a counter argument to this conclusion. Here, we show that these authors are merely repeating the conventional mistake of incorrectly placing the observer simultaneously in a comoving frame, where the lapse function $g_{t t}$ is coordinate dependent when $\rho+3 p \neq 0$, and a supposedly different, free-falling frame, in which $g_{t t}=1$, implying no time dilation. We demonstrate that the Hubble flow is not inertial when $\rho+3 p \neq 0$, so the comoving frame is generally not in free fall, even though in FRW, the comoving and free-falling frames are supposed to be identical at every spacetime point. So this confusion of frames not only constitutes an inconsistency with the fundamental tenets of general relativity but, additionally, there is no possibility of using a gauge transformation to select a set of coordinates for which $g_{t t}=1$ when $\rho+3 p \neq 0$.
\end{abstract}

Keywords: cosmology; gravitation; spacetime metric

PACS numbers: 04.20.Ex, 95.36.+x, 98.80.-k, 98.80.Jk

*John Woodruff Simpson Fellow; Electronic address: fmelia@email.arizona.edu 


\section{INTRODUCTION}

The standard model of cosmology $(\Lambda \mathrm{CDM})$ is quite successful in accounting for many observations, in part due to its rather large number of free parameters. These include the spatial curvature constant $k$, the Hubble constant $H_{0}$ and the scaled matter $\left(\Omega_{\mathrm{m}}\right)$, radiation $\left(\Omega_{\mathrm{r}}\right)$, and dark-energy $\left(\Omega_{\mathrm{de}}\right)$ densities. In terms of the critical density $\rho_{\mathrm{c}} \equiv 3 c^{2} H_{0}{ }^{2} / 8 \pi G$, one writes $\Omega_{i} \equiv \rho_{i} / \rho_{\mathrm{c}}$, where $i$ represents the energy density $\rho_{\mathrm{m}}, \rho_{\mathrm{r}}$, or dark energy $\rho_{\mathrm{de}}$, as the case may be. Without invoking priors, one must typically optimize at least five unspecified parameters, all of which can be adjusted to fit the data.

Given this wide latitude of possible outcomes for the expansion history of the Universe, it is therefore very surprising to see that the optimization of model parameters, especially using anisotropies in the cosmic microwave background (CMB) [1-3], reveals a universal expansion with an average acceleration of zero (within the measurement errors) over a Hubble time $H_{0}^{-1}[4-8]$. Another way to characterize this empirical result, in terms of the total pressure $p=p_{\mathrm{r}}+p_{\mathrm{m}}+p_{\mathrm{de}}$ and energy density $\rho=\rho_{\mathrm{r}}+\rho_{\mathrm{m}}+\rho_{\mathrm{de}}$, is to note that averaged over a Hubble time, the quantity $\langle p / \rho\rangle$ has the value $-1 / 3$ just at this moment, when we happen to be looking. Yet the combination of $\rho_{\mathrm{m}}, \rho_{\mathrm{r}}$ and $\rho_{\mathrm{de}}$ could have produced a wide assortment of epochs with deceleration and acceleration.

This outcome is not merely surprising [9]. In the context of $\Lambda \mathrm{CDM}$, the condition $\langle p / \rho\rangle=$ $-1 / 3$ can be achieved only once in the entire (presumably infinite) history of the Universe, making it astonishingly unlikely. There is clearly physics behind this zero active mass condition. In a recent paper [8], we showed that the Friedmann-Robertson-Walker (FRW) metric is in fact valid only for a cosmic fluid with an equation of state (EoS) $\rho+3 p=0$, so the condition $\langle p / \rho\rangle=-1 / 3$ is independent of time. In other words, no matter how imperfect or incomplete our parametrization of the standard model happens to be, the optimized parameter values must always yield a zero average acceleration, regardless of when we make the measurements.

Kim et al. [9] have challenged this conclusion by providing what they believe to be a counter argument to the zero active mass condition in FRW. In this paper, however, we demonstrate that their claim is based on the conventional mistake of writing the metric from the perspetive of an observer who is simultaneously in a non-inertial comoving frame, where a time dilation is unavoidable for an accelerated expansion of the spatial coordinates, 
and in a different, free-falling frame, with no measurable acceleration, thereby "assigning" without justification a constant value of one to the lapse function $g_{t t}$.

\section{THE LAPSE FUNCTION IN FRW}

With the adoption of the Cosmological principle, the spacetime metric may be simplified to the FRW form

$$
d s^{2}=c^{2} d t^{2}-a^{2}(t)\left[d r^{2}\left(1-k r^{2}\right)^{-1}+r^{2}\left(d \theta^{2}+\sin ^{2} \theta d \phi^{2}\right)\right],
$$

in terms of the cosmic time $t$, the comoving radius $r$, the universal expansion factor $a(t)$, and the angular coordinates $\theta$ and $\phi$ in the comoving frame. The spatial curvature constant $k$ takes on the values $(-1,0,+1)$, for an open, flat, or closed universe, respectively.

Two rarely discussed issues with this metric are (1) that its lapse function $g_{t t}$ is constant, and (2) that in deriving its coefficients, one never considers whether the selected components of the energy-momentum tensor $T^{\mu \nu}$ are consistent with the assumption of homogeneity and isotropy. In general relativity (GR), a constant lapse function arises only in a free-falling frame, where the observer is not subject to any gravitational influences. So even before we discuss the formal relationship between $g_{t t}$ and $T^{\mu \nu}$, proponents of the use of Equation (1) to describe the spacetime of an accelerating Universe need to explain why this basic tenet of relativity theory should be violated, i.e., why in the case of FRW an observer in the free-falling frame nonetheless sees a gravitational acceleration without a corresponding time dilation.

The general class of spherically-symmetric spacetime metrics, of which FRW is a special case, may be represented as

$$
d s^{2}=e^{2 \Phi / c^{2}} c^{2} d t^{2}-e^{\lambda} d r^{2}-R^{2} d \Omega^{2}
$$

where $d \Omega^{2} \equiv d \theta^{2}+\sin ^{2} \theta d \phi^{2}$, and $\Phi, \lambda$, and $R$ are each functions of $r$ and $t$, and are to be determined by solving Einstein's equations. It is somewhat tedious, though straightforward, to derive the dependence of $g_{t t} \equiv e^{2 \Phi / c^{2}}$ on $T^{t t}, T^{r r}, T^{\theta \theta}$ and $T^{\phi \phi}$. In the conventional approach, however, $\Phi$ is set equal to 0 to arrive at Equation (1) without following this procedure. As such, the FRW form of the metric does not produce any time dilation relative to the proper time in a local inertial frame, even in cases where the observer sees an accelerated 
expansion of the spatial coordinates, i.e., when $\ddot{a} \neq 0$. This is critical because, as we shall see shortly, the Hubble flow in FRW is not inertial when $\ddot{a} \neq 0$.

But as shown in ref. [8], $g_{t t}$ is in fact not equal to one when $\rho+3 p \neq 0$, a requirement of $\ddot{a} \neq 0$. Borrowing a result from that paper, we find that

$$
e^{2 \Phi(t) / c^{2}}=h \dot{a}^{2} e^{\mathcal{I}(t)}
$$

where

$$
\mathcal{I}(t) \equiv \int_{0}^{t} d t^{\prime} \frac{8 \pi G}{3 c^{2} H} e^{\Phi / c^{2}}(\rho+3 p)
$$

and $H \equiv e^{-\Phi / c^{2}}(\dot{a} / a)$ is the Hubble constant. This expression provides the necessary, formal relationship between the lapse function $g_{t t}$ and the active mass $\rho+3 p$ as seen by an observer in the comoving frame. Clearly, $\Phi$ changes for different values of the active mass and corresponding expansion history encoded into the integral for $\mathcal{I}$ over cosmic time. In order to achieve a constant $\Phi$, we must have $\mathcal{I} \rightarrow 0$, which is guaranteed only when $\rho+3 p \rightarrow 0$. Then one can show that $\dot{a}$ is also constant, and we may set $g_{t t}=1$ with an appropriate choice of the initial condition $h$.

This is where Kim, Lasenby and Hobson [9] interject with a counter claim that one is free to choose $\Phi=0$, in spite of its evident dependence on $\rho+3 p$. They do so without explaining why they view Equation (3) as allowing $\Phi$ to have different values for the same $\rho+3 p$, and without providing a physical justification for making their particular choice leading to $g_{t t}=1$. In this regard, their approach is no different from the conventional procedure of simply forcing $g_{t t}=1$ in Equation (1) before the metric is introduced into Einstein's equations, thereby removing any possibility of a time dilation. The answer, of course, is that for the same $\rho+3 p$, different values of $\Phi$ correspond to different coordinate systems. And in order to force $\Phi=0$, while keeping $\mathcal{I}(t) \neq 0$, they must consider the observer to be simultaneously in the comoving frame where an active mass $\rho+3 p \neq 0$ produces acceleration (according to the second Friedmann equation, in which $\ddot{a} \sim \rho+3 p$ ) and $\Phi \neq 0$, and in the free-falling frame where $g_{t t}=1$ by choice. If the introduction of the spherically-symmetric form of the metric (Equation 2) into Einstein's equations were to reduce to the FRW spacetime shown in Equation (1) irrespective of how one chooses the active mass, then this should happen automatically and unambiguously, without the manual intervention by Kim et al. [9] to force $\Phi=0$ in Equation (3). As we shall see below, this 
automatic reduction to Equation (1) does not happen because the comoving frame is not inertial, except in the special case when $\rho+3 p=0$.

This is also the reason why a gauge transformation cannot be used to eliminate the coordinate-dependent $g_{t t}$ after the fact. Often misunderstood and incorrectly used, a gauge transformation in GR is a transformation of the coordinates selected in order to alter the metric coefficients $g_{\mu \nu}$ to a desired form. Since $\Phi$ is solely a function of $t$, it is straightforward to find the necessary transformation for FRW but, as discussed in ref. [8], such a transformation would require the existence of two distinct frames of reference: one (the comoving) frame in which the observer sees $\ddot{a} \neq 0$ when $\rho+3 p \neq 0$, and therefore $g_{t t} \neq 1$, and a second (free-falling) frame in which the observer sees no time dilation and no acceleration. In other words, an observer cannot carry out an actual gauge transformation and stay within the same frame. And since the comoving frame in FRW is generally not inertial, an observer who experiences gravitational effects that lead to a coordinate-dependent lapse function cannot simultaneously also be in a free-falling frame where he does not measure a time dilation.

Ultimately, the issue we have been discussing here, and in refs. [8,9], has to do with whether or not one can always clearly distinguish between accelerated and inertial frames. The answer is yes (see, e.g., ref. [10], § 3.2). In general relativity, acceleration can always be measured absolutely, unlike velocity, which is only measurable in a relative sense. For this reason, we can always find a local diffeomorphism that reduces the chosen manifold's metric to a Minkowski metric in a sufficiently small neighborhood of a given spacetime point when tidal forces are ignored $[11,12]$. The FRW metric is not asymptotically flat and has a non-vanishing spacetime curvature tensor $R_{\alpha \beta \gamma \delta}$. Thus, in spite of the fact that its Weyl tensor is zero, which allows Equation (1) to be written as a conformally-flat metric, the comoving frame used to write the FRW metric is not in free fall.

One seldom sees this property of FRW invoked in the interpretation of measurements made by a Hubble observer (however, see, refs. $[13,14,15]$ ), but one can easily demonstrate that the Hubble flow in FRW is in fact not inertial when $\rho+3 p \neq 0$. The metric coefficients $g_{\mu \nu}(x)$ and affine connections $\Gamma_{\mu \nu}^{\lambda}(x)$ at $x^{\alpha}=(c t, r, \theta, \phi)$ contain enough information for us to determine the local inertial coordinates $\xi^{\alpha}(x)$ in the neighborhood of $x^{\alpha}$. As shown, e.g., 
in ref. $[10]$ or $[13,14]$, the local inertial coordinates $\xi^{\alpha}(x)$ satisfy the equation

$$
\frac{\partial^{2} \xi^{\mu}}{\partial x^{\lambda} \partial x^{\kappa}}=\Gamma_{\lambda \kappa}^{\nu}(x) \frac{\partial \xi^{\mu}}{\partial x^{\nu}}
$$

In their most general form, the non-vanishing affine connections in FRW are

$$
\begin{array}{llll}
\Gamma_{00}^{0}=\dot{\Phi} / c^{3} & & \\
\Gamma_{r r}^{0}=\frac{1}{c} a \dot{a} e^{-2 \Phi / c^{2}} & \Gamma_{\theta \theta}^{0}=\frac{1}{c} a \dot{a} r^{2} e^{-2 \Phi / c^{2}} & \Gamma_{\phi \phi}^{0}=\frac{1}{c} a \dot{a} r^{2} \sin ^{2} \theta e^{-2 \Phi / c^{2}} \\
\Gamma_{r 0}^{r}=\frac{1}{c} \frac{\dot{a}}{a} & \Gamma_{\theta \theta}^{r}=-r & \Gamma_{\phi \phi}^{r}=-r \sin ^{2} \theta \\
\Gamma_{\theta 0}^{\theta}=\frac{1}{c} \frac{\dot{a}}{a} & \Gamma_{\theta r}^{\theta}=\frac{1}{r} & \Gamma_{\phi \phi}^{\theta}=-\cos \theta \sin \theta \\
\Gamma_{\phi 0}^{\phi}=\frac{1}{c} \frac{\dot{a}}{a} & \Gamma_{\phi r}^{\phi}=\frac{1}{r} & \Gamma_{\phi \theta}^{\phi}=\cot \theta .
\end{array}
$$

Introducing these into Equation (5), we see that the inertial coordinates $\xi^{\mu}=(c \tilde{t}, \tilde{r}, \tilde{\theta}, \tilde{\phi})$ must satisfy the following expressions:

$$
\begin{gathered}
\frac{\partial^{2} \tilde{t}}{\partial t^{2}}=\frac{\dot{\Phi}}{c^{2}} \frac{\partial \tilde{t}}{t} \\
\frac{\partial^{2} \tilde{t}}{\partial t \partial r}=\frac{\dot{a}}{a} \frac{\partial \tilde{t}}{\partial r} \\
\frac{\partial^{2} \tilde{t}}{\partial r^{2}}=\frac{1}{c^{2}} \dot{a} a e^{-2 \Phi / c^{2}} \frac{\partial \tilde{t}}{\partial t} \\
\frac{\partial^{2} \tilde{r}}{\partial t^{2}}=\frac{\dot{\Phi}}{c^{2}} \frac{\partial \tilde{r}}{\partial t}
\end{gathered}
$$

and

$$
\frac{\partial^{2} \tilde{r}}{\partial r \partial t}=\frac{1}{c} \frac{\dot{a}}{a} \frac{\partial \tilde{r}}{\partial r}
$$

Let us first consider the local inertial frame for an FRW cosmology with zero active mass (i.e., $\rho+3 p=0$ ), corresponding to $a(t)=t / t_{0}$ (normalized such that $a=1$ today). We will place the observer near the origin of his coordinates, so that $r / c t \ll 1$. For this equation of state, we also have $\Phi=0$ (see Equation 3). The solution to Equations (6-10) is

$$
\tilde{r}=a(t) r
$$

and

$$
\tilde{t} \approx t\left(1+\frac{1}{2}\left[\frac{\tilde{r}}{c t}\right]^{2}\right),
$$

correct to second order in $r / c t$. Thus, according to these expressions, the local inertial frame in the vicinity of $r=0$ coincides with the Hubble flow and, in this situation, one has 
$\ddot{a}=0$ with $g_{t t}=1$. Thus, for this case (and this case only), it is legitimate to consider the comoving and free-falling frames to be identical, and for the observer to see no acceleration and no time dilation, so that $\Phi=0$.

When $\rho+3 p \neq 0$, however, $a(t)$ is no longer linear in $t$, and $\tilde{r}$ in Equation (11) no longer satisfies Equations (9) and (10), regardless of whether or not one uses $\Phi=0$. For example, in order for $\tilde{r}=$ ar to be consistent with Equation (9), we would need either $\ddot{a}=0$ (if $\Phi=0$ ), or $\ddot{a}-\dot{a} \dot{\Phi} / c=0$ (if $\Phi \neq 0$ ), both of which occur only when [8] $\rho+3 p=0$. Of course, one can still find a local inertial frame when $\rho+3 p \neq 0$, but it is not coincident with the Hubble flow [11-15]. In this instance, the solution may be written as a polynomial to second order in $r$, as demonstrated in $\S 3.2$ of ref. [10], or [13,14], but we don't need to reproduce its coefficients here. The fact that the Hubble flow in FRW is not an inertial frame when $\rho+3 p \neq 0$ is sufficient to prove our argument. Therefore, setting $\Phi=0$ when $\rho+3 p \neq 0$ in the comoving frame is not consistent with the fact that a time dilation should be measurable as a result of the acceleration seen by the observer.

The confusion about the measurability of a time dilation due to acceleration in FRW may be due to the fact that, because of homogeneity, $g_{t t}$ can only be a function of $t$, not $r$, unlike the situation in the Schwarzschild spacetime, where it is a function of $r$ and not of $t$. But this difference in coordinates does not change the requirement that an acceleration must always produce a time dilation that is measurable relative to the passage of proper time in an inertial frame. Therefore, regardless of whether $g_{t t}$ is a function of $t$ or $r$, the time dilation resulting from an accelerated expansion of the spatial coordinates cannot be "hidden" from the observer. Putting $g_{t t}=1$ in Equation (1), even when $\ddot{a} \neq 0$, suggests otherwise, and is therefore inconsistent with basic relativity theory. To guarantee that $\Phi=0$ in Equation (3), one must have $\rho+3 p=0$.

Concerned by "gauge ambiguities" in their first attempt at challenging the zero active mass condition, Kim et al. [16] extended their argument by attempting to derive the FRW solution using a tetrad, which they claim constitutes a superior method that avoids such gauge ambiguities. They apparently believe that the physics of a problem may be changed merely by altering the calculational technique. But this is clearly false; if a gauge amibuity is present with one approach, it is present for all approaches because, as we have emphasized all along, a gauge transformation is not arbitrary — it constitutes a transformation of coordinates from one frame to another. Kim et al. believe that the sophistication of the tetrad approach 
somehow selects a unique frame of reference, creating their sought-after merger of a noninertial comoving frame with a free-falling frame. This thinking, however, is flawed. The tetrad and its inverse simply package the metric coefficients and, once a solution is found, they must be unpacked to restore the spacetime coordinates. One therefore gets out what one puts in. In this regard, their approach is similar to that of Tupper [17], who explicitly set $g_{t t}=1$ in his use of the tetrad field equations to derive a generalized Friedmann equation. Kim et al.'s use of the tetrad is more subtle, but they too invoked a special choice of coordinates - specifically, a particular choice of time - in order to simplify their tetrad ansatz before using the field equations to derive a solution.

Finally, we briefly comment on Kim et al.'s attempt to discredit the role of $R_{\mathrm{h}}$ as a true gravitational horizon. In so doing, they follow the lead of van Oirschot et al. [18] and Lewis et al. [19], who failed to realize that a horizon is observer dependent. An observer "sees" a horizon only in terms of null geodesics that actually reach him. Thus, to correctly interpret the role played by the surface at $R_{\mathrm{h}}$, one must actually solve the null geodesic equations, as demonstrated by Bikwa et al. [20] and Melia [21], not simply rely on how or when $R_{\mathrm{h}}$ changes with time. A complete formal discussion of how $R_{\mathrm{h}}$ delimits the portion of the Universe visible to us today is given in Melia [22].

\section{CONCLUDING REMARKS}

In this paper we have shown that the counter argument raised by Kim, Lasenby and Hobson [9] is simply based on the conventional approach of forcing the lapse function of the metric to be constant, without providing a physical justification for this particular "choice," even when the formal relationship between $g_{t t}=e^{2 \Phi / c^{2}}$ and $T^{\mu \nu}$ permits a range of possibilities for the same active mass $\rho+3 p$. In reality, a specific selection of $\Phi$ corresponds to a particular choice of coordinates. In FRW, the Hubble flow is non-inertial when $\rho+3 p \neq 0$, so the comoving frame cannot be identical to the free-falling frame. Therefore one cannot measure an acceleration without a corresponding time dilation.

Acknowledgements: I am grateful to PMO in Nanjing, China, for its hospitality while this work was being carried out. This work was partially supported by grant 2012T1J0011 from The Chinese Academy of Sciences Visiting Professorships for Senior International Scientists, and grant GDJ20120491013 from the Chinese State Administration of Foreign Experts Af- 
fairs.

[1] C. L. Bennett et al., ApJS 148, 97 (2003).

[2] D. N. Spergel et al., ApJS 148, 175 (2003).

[3] P.A.R. Ade et al., A\&A 571, od A23 (2014).

[4] F. Melia The Edge of Infinity: Supermassive Black Holes in the Universe (Cambridge Univ Press, Cambridge), p. 119 (1972).

[5] F. Melia, MNRAS 382, 1917 (2007).

[6] F. Melia and M. Abdelqader, IJMP-D 18, 1889 (2009).

[7] F. Melia and A.S.H. Shevchuk, MNRAS 419, 2579 (2012).

[8] F. Melia, Front Phys 11, id 119801 (2016).

[9] D. Y. Kim, A. N. Lasenby and M. P. Hobson, MNRAS submitted (2016) [arXiv:1601.07890].

[10] S. Weinberg, Gravitation and Cosmology: Principles and Applications of the General Theory of Relativity (Wiley, New York) (1972).

[11] A. Harvey, Annals of Physics 29(3), 383 (1964).

[12] M. Carrera and D. Giulin,i Reviews of Modern Physics 82, 169 (2010).

[13] H. Liu, J Math Phys 28, 1920 (1987).

[14] H. Liu, J Math Phys 28, 1924 (1987).

[15] S. M. Kopeikin, Eur Phys J Plus 130, 11 (2015).

[16] D. Y. Kim, A. N. Lasenby and M. P. Hobson, PRD submitted (2016) [arXiv:1604.06365].

[17] B.O.J. Tupper, Astrophys. Sp. Sci. 28, 225 (1974).

[18] P. van Oirschot, J. Kwan and G. F. Lewis, MNRAS 404, 1633 (2010).

[19] G. F. Lewis and P. van Oirschot, MNRAS Letters 423, 26 (2012).

[20] O. Bikwa, F. Melia and A.S.H. Shevchuk, MNRAS 421, 3356 (2012).

[21] F. Melia, JCAP 09, 029 (2012).

[22] F. Mela, CQG 30, 155007 (2013). 